\title{
COMPLEX ALMOST CONTACT STRUCTURES IN A COMPLEX CONTACT MANIFOLD
}

\author{
By SHIgERU ISHIHARA AND MARIKo Konishi
}

\section{$\S 1$. Introduction}

Let $M$ be a complex manifold of odd dimension $2 m+1(\geqq 3)$ covered by an open covering $\mathfrak{A}=\left\{O_{i}\right\}$ consisting of coordinate neighborhoods. If there is a holomorphic 1 -form $\omega_{i}$ in each $O_{i} \in \mathfrak{A}$ in such a way that for any $O_{\imath}, O_{j} \in \mathfrak{A}$

$$
\begin{array}{ll}
\omega_{i} \wedge\left(d \omega_{i}\right)^{m} \neq 0 & \text { in } \quad O_{\imath}, \\
\omega_{i}=f_{\imath \jmath} \omega_{j} & \text { in } \quad O_{i} \cap O_{j} \neq \phi,
\end{array}
$$

where $f_{\imath \jmath}$ is a holomorphic function in $O_{i} \cap O_{\jmath}$, then the set $\left\{\left(\omega_{i}, O_{2}\right) \mid O_{i} \in \mathfrak{A}\right\}$ of local structures is called a complex contact structure and $M$ a complex contact manifold, where $\omega_{i}$ is called the contact form in $O_{\imath}$.

On the other hand, suppose that there are given in each $O_{i} \in \mathfrak{A}$ a 1 -form $u_{\imath}$, a vector field $U_{\imath}$ and a tensor field $G_{\imath}$ of type $(1,1)$ satisfying the following condition $(1.2, \mathrm{i})$ and $(1.2, \mathrm{ii})$ : for any $O_{\imath}, O_{j} \in \mathfrak{A}$

$$
\begin{gathered}
G_{\imath}{ }^{2}=-I+u_{i} \otimes U_{\imath}+v_{\imath} \otimes V_{\imath}, \quad G_{\imath} F=-F G_{\imath}, \\
u_{i} \circ G_{\imath}=0, \quad u_{\imath}\left(U_{i}\right)=1,
\end{gathered}
$$

$I$ and $F$ being respectively the identity tensor field of type $(1,1)$ and the complex structure of $M$, where $v_{i}$ and $V_{\imath}$ are defined in $O_{\imath}$ respectively by

$$
v_{\imath}=u_{i} \circ F, \quad V_{\imath}=-F U_{\imath} .
$$

In $O_{i} \cap O_{j} \neq \phi$ there are functions $a$ and $b$ in such a way that

$$
\begin{array}{ll}
u_{\imath}=a u_{\jmath}-b v_{\jmath}, & G_{\imath}=a G_{j}-b H_{\jmath}, \\
v_{i}=b u_{j}+a v_{\jmath}, & H_{\imath}=b G_{\jmath}+a H_{\jmath},
\end{array} \quad \text { in } O_{i} \cap O_{\jmath},
$$

where $H_{\imath}$ is defined in $\mathrm{O}_{\imath}$ by

$$
H_{\imath}=F G_{\imath} .
$$

Then the set $\left\{\left(u_{\imath}, U_{\imath}, G_{\imath}, O_{\imath}\right) \mid O_{i} \in \mathfrak{X}\right\}$ of local structures is called a complex

Received June 30, 1980. 
almost contact structure and $M$ a complex almost contact manifold.

It is the purpose of the present paper to discuss the relation between complex contact structures and complex almost contact structures and to prove

THEOREM Let $M$ be a complex contact manifold of odd complex dimension $2 m+1$ ( $\geqq 3)$. Then $M$ admits always a complex almost contact structure of class $C^{\infty}$.

This subject has been partially studied in [3] and proved that a complex almost contact manifold admits a complex contact structure if it is normal.

\section{$\S 2$. Lemmas.}

From now on, $M$ is assumed to be a complex contact manifold of complex dimension $2 m+1(\geqq 3)$ with structure $\left\{\left(\omega_{i}, O_{\imath}\right) \mid O_{i} \in \mathfrak{A}\right\}$. Then for any $O_{\imath}, O_{\jmath}, O_{k}$ $\in \mathfrak{A}$

$$
\omega_{i}=f_{\imath \jmath} \omega_{j} \quad \text { in } \quad O_{i} \cap O_{\jmath} \neq \phi
$$

and hence the cocycle condition

$$
f_{\jmath k} f_{k \imath} f_{\imath \jmath}=1 \quad \text { in } \quad O_{\imath} \cap O_{\jmath} \cap O_{k}
$$

holds. Then there is a complex line bundle $\widetilde{P}$ over $M$ with $\left\{f_{\imath j}\right\}$ as its transition functions. Denote by $P$ a circle bundle over $M$ associated with $\tilde{P}$. Then there is a non-vanishing complex-valued function $\tau_{\imath}$ in $O_{i} \in \mathfrak{A}$ such that the function

$$
h_{\imath \jmath}=\tau_{\imath}^{-1} f_{\imath \jmath} \tau_{\jmath} \quad \text { in } \quad O_{i} \cap O_{\jmath} \neq \phi
$$

is the transition function of $P$ in $O_{i} \cap O$, and satisfies the condition

On putting

$$
\left|h_{\imath j}\right|=1 \text {. }
$$

$$
\pi_{\imath}=\tau_{\imath}^{-1} \omega_{i} \quad \text { in } O_{\imath},
$$

we have by using (2.1) and (2.2)

$$
\pi_{\imath}=h_{\imath \jmath} \pi_{\jmath}, \quad\left|h_{\imath \jmath}\right|=1 \text { in } O_{\imath} \cap O_{\jmath} .
$$

Let $\sigma$ be a connection in the circle bundle $P$. Then there is a local real 1 -form $\sigma_{\imath}$ in each $O_{\imath} \in \mathfrak{A}$ such that

$$
\sqrt{ }-1 \sigma_{\imath}=\sqrt{ }-1 \sigma_{\jmath}+\frac{d h_{\imath \jmath}}{h_{\imath \jmath}} \quad \text { in } \quad O_{i} \cap O_{\jmath} .
$$

The condition $\omega_{\imath} \wedge\left(d \omega_{i}\right)^{m} \neq 0$ implies

$$
\pi_{\imath} \wedge\left(d \pi_{\imath}\right)^{m} \neq 0 \quad \text { in } \quad O_{\imath}
$$

and hence 


$$
\pi_{\imath} \wedge \Omega_{\imath}{ }^{m} \neq 0 \quad \text { in } O_{\imath},
$$

where $\Omega_{\imath}$ is defined by

$$
\Omega_{\imath}=d \pi_{i}-\sqrt{-1} \sigma_{\imath} \wedge \pi_{\imath} \text { in } O_{\imath} .
$$

Thus $\Omega_{\imath}$ is a local 2 -form of rank $2 m$ in each $O_{\imath}$ and

$$
\Omega_{\imath}=h_{\imath j} \Omega_{\jmath} \quad \text { in } \quad O_{i} \cap O_{\jmath}
$$

holds because of (2.3) and (2.4). Thus we have

LEMMA 2.1. There is in each $O_{i} \in \mathfrak{A}$ a 2-form $\Omega_{\imath}$ of rank $2 \mathrm{~m}$ and of class $C^{\infty}$ satısfying (2.8).

We now put

$$
u_{\imath}=\frac{1}{2}\left(\pi_{i}+\bar{\pi}_{\imath}\right), \quad v_{\imath}=\frac{1}{2 \sqrt{-1}}\left(\pi_{\imath}-\bar{\pi}_{\imath}\right)
$$

which are local real 1 -forms in each $O_{\imath} \in \mathfrak{A}$. Then we obtain

$$
v_{i}=u_{i} \circ F,
$$

$F$ being the complex structure of $M$. Using (2.5), we get

$$
\begin{aligned}
& u_{\imath}=a u_{j}-b v_{\jmath}, \\
& v_{i}=b u_{j}+a v_{\jmath},
\end{aligned}
$$

where $h_{\imath \jmath}=a+\sqrt{-1} b$. The relation (2.5) implies

$$
\left(\pi_{i}+\bar{\pi}_{\imath}\right) \wedge\left(d \pi_{i}+d \bar{\pi}_{\imath}\right)^{m} \neq 0, \quad\left(\pi_{\imath}-\bar{\pi}_{\imath}\right) \wedge\left(d \pi_{\imath}-d \bar{\pi}_{\imath}\right)^{m} \neq 0,
$$

or equivalently

$$
u_{\imath} \wedge v_{i} \wedge\left(d u_{\imath}\right)^{2 m} \neq 0, \quad u_{\imath} \wedge v_{i} \wedge\left(d v_{i}\right)^{2 m} \neq 0 .
$$

On the other hand, we obtain from (2.7) and (2.9)

$$
\begin{aligned}
& \frac{1}{2}\left(\Omega_{i}+\bar{\Omega}_{\imath}\right)=d u_{i}-\sigma_{\imath} \wedge v_{i}, \\
& \frac{1}{2 \sqrt{ }-\overline{1}}\left(\Omega_{i}-\bar{\Omega}_{\imath}\right)=d v_{i}+\sigma_{\imath} \wedge u_{\imath} .
\end{aligned}
$$

Therefore (2.12) and (2.13) imply

$$
u_{\imath} \wedge v_{i} \wedge\left(\hat{G}_{i}\right)^{2 m} \neq 0, \quad u_{\imath} \wedge v_{i} \wedge\left(\hat{H}_{\imath}\right)^{2 m} \neq 0,
$$

where

$$
\hat{G}_{i}=d u_{i}-\sigma_{\imath} \wedge v_{i}, \quad \hat{H}_{\imath}=d v_{i}+\sigma_{\imath} \wedge u_{\imath} .
$$

Thus (2.8), (2.11), (2.12) and (2.13) imply 
LEMMA 2.2. There are in each $O_{i} \in \mathfrak{A}$ skew-symmetruc local tensor fields $\hat{G}_{2}$ and $\hat{H}_{\imath}$ of rank $4 \mathrm{~m}$ such that

$$
\hat{H}_{i}(X, Y)=\hat{G}_{i}(F X, Y), \quad \hat{G}_{i}(X, Y)=-\hat{H}_{\imath}(F X, Y) \quad \text { in } \quad O_{\imath}
$$

for any vector fields $X$ and $Y$ and

$$
\hat{G}_{i}=a \hat{G}_{\jmath}-b \hat{H}_{\jmath}, \quad \hat{H}_{2}=b \hat{G}_{j}+a \hat{H}_{\jmath}, \quad \text { in } \quad O_{i} \cap O_{\jmath},
$$

where $h_{\imath \jmath}=a+\sqrt{-1} b$.

We now state two more lemmas which are essential in the proof of our theorem (cf. [1], [2], [4]). Let $O(n)$ be the orthogonal group acting on $n$ variables, $H(n)$ be the space consisting of all positive definite symmetric $(n, n)$ matrices and $G L(n, \boldsymbol{R})$ be the general linear group acting on $n$ variables.

LEMMA 2.3. Any real non-singular $(n, n)$-matrix $\rho$ can be written in one and only one way as the product $\rho=\alpha \beta$ with $\alpha \in O(n)$ and $\beta \in H(n)$. The mapping $\phi: G L(n, \boldsymbol{R}) \rightarrow O(n) \times H(n)$ defined by this decomposition gives a homeomorphism.

Remark. Since $O(n)$ and $H(n)$ are real analytic submanifolds of $G L(n, \boldsymbol{R})$, $\phi: G L(n, \boldsymbol{R}) \rightarrow O(n) \times H(n)$ is a real analytic homeomorphism, i.e. any $\rho \in$ $G L(n, \boldsymbol{R})$ can be decomposed analytically in one and only one way as $\rho=\alpha \beta$, where $\alpha \in O(n)$ and $\beta \in H(n)$, (See Hatakeyama [2] for example).

LEMMA 2.4. Let $\mathfrak{A}=\left\{O_{i}\right\}$ be an open covering of $M$ by coordinate nerghborhoods. Suppose that there is in each $O_{i} \in \mathfrak{U}$ a local tensor fields $\alpha_{\imath}$ of type (0.2) and of class $C^{\infty}$. Choose a field of orthonormal frames in each $O_{i} \in \mathfrak{A}$ and let $\gamma_{i}$ be the transformations of these fields of orthonormal frames in $O_{i} \cap O_{\jmath}$. Then $\left\{\alpha_{i}\right\}$ defines globally a tensor field of class $C^{\infty}$ in $M$ if and only if $\gamma_{\imath \jmath} \alpha_{\jmath}{ }^{t} \gamma_{\imath \jmath}$ $=\alpha_{\imath}$ holds in $O_{i} \cap O_{\jmath}$ for any $O_{\imath}, O_{j} \in \mathfrak{A}$.

\section{$\S 3$. Proof of theorem}

Let $\hat{G}_{i}$ and $\hat{H}_{\imath}$ be the skew-symmetric tensors appearing in Lemma 2.2. If we put for any $p \in O_{\imath}(\in \mathfrak{U})$

$$
D_{i}(p)=\left\{Y \in T_{p}\left(O_{\imath}\right): \hat{G}_{i}(Y, X)=0 \text { for any } X \in T_{p}\left(O_{\imath}\right)\right\},
$$

then we get in $O_{\imath}$ a local distribution $D_{\imath}: p \mapsto D_{\imath}(p)$. Lemma 2.2 implies $D_{\imath}(p)$ $=D_{\jmath}(p)$ for any $p \in O_{i} \cap O_{\jmath}$. Hence the local distributions $D_{\imath}$ defined in $O_{\imath}$ determines a global distribution $D$ in $M$, which is of real dimension 2 .

LEMMA 3.1. There is a unique local basis $\left\{U_{\imath}, V_{i}\right\}$ of the distribution $D$ in each $O_{i} \in \mathfrak{A}$ such that

$$
\begin{aligned}
& u_{\imath}\left(U_{\imath}\right)=1, \quad u_{\imath}\left(V_{\imath}\right)=0, \quad v_{\imath}\left(U_{\imath}\right)=0, \quad v_{\imath}\left(V_{\imath}\right)=1, \\
& \hat{G}_{i}\left(U_{\imath}, X\right)=\hat{G}_{i}\left(V_{\imath}, X\right)=\hat{H}_{i}\left(U_{\imath}, X\right)=\hat{H}_{\imath}\left(V_{\imath}, X\right)=0,
\end{aligned}
$$


for any vector field $X$ and

$$
U_{\imath}=a U_{j}-b V_{\jmath}, \quad V_{\imath}=b U_{j}+a V_{\jmath} \quad \text { in } \quad O_{i} \cap O_{\jmath} .
$$

Proof. If we put for any $p \in O_{\imath}(\in \mathfrak{U})$

$$
A_{\imath}(p)=\left\{Y \in T_{p}\left(O_{\imath}\right): v_{\imath}(Y)=0\right\},
$$

then we get a distribution $A_{\imath}: p \mapsto A_{i}(p)$ in $O_{\imath}$. The distribution $D \cap A_{2}$ is 1dimensional in $O_{\imath}$ because of (2.14). Thus there is a unique vector field $U_{\imath}$ in $O_{\imath}$ such that $U_{\imath}$ spans $D \cap A_{\imath}$ and $u_{\imath}\left(U_{\imath}\right)=1$, i. e. such that

$$
\hat{G}_{i}\left(U_{\imath}, X\right)=0, \quad u_{i}\left(U_{\imath}\right)=1, \quad v_{i}\left(U_{\imath}\right)=0 \quad \text { in } O_{\imath}
$$

for any vector field $X$. Lemma 2.2 implies

$$
\hat{H}_{i}\left(U_{\imath}, X\right)=0
$$

for any vector field $X$. Putting in $O_{\imath}$

$$
V_{\imath}=-F U_{\imath}
$$

and using Lemma 2.2 and (2.10), we get

$$
\hat{G}_{i}\left(V_{\imath}, X\right)=\hat{H}_{\imath}\left(V_{\imath}, X\right)=0, \quad v_{i}\left(V_{\imath}\right)=1, \quad u_{i}\left(V_{\imath}\right)=0
$$

for any vector field $X$. Therefore $\left\{U_{\imath}, V_{i}\right\}$ is in $O_{\imath}$ a local basis of the distribution $D$. As a consequence of (2.11), we have

$$
U_{\imath}=a U_{\jmath}-b V_{\jmath}, \quad V_{\imath}=b U_{j}+a V_{\jmath}, \quad \text { in } O_{i} \cap O_{\jmath} .
$$

Thus Lemma 3.1 is proved.

We shall now prove the theorem stated in $\S 1$.

Proof of theorem. Let $\tilde{g}$ be a Hermitian metric in $M$ such that $\tilde{g}\left(U_{\imath}, X\right)$ $=u_{\imath}(X)$ and $\tilde{g}\left(V_{\imath}, X\right)=v_{\imath}(X)$ for any vector field $X$. Take an orthonormal adapted frame $\left\{E_{1}, F E_{1}, \cdots, E_{2 m}, F E_{2 m}, U_{\imath}, V_{i}\right\}$ with respect to $g$ in each $O_{i} \in \mathfrak{A}$. Then by Lemma $3.1 \hat{G}_{i}$ has components of the form

$$
\Phi_{\imath}=\left(\begin{array}{cccc}
\Phi_{\imath}^{\prime} & \vdots & & 0 \\
\cdots \cdots \cdots & \ldots \ldots \ldots \ldots \\
& \vdots & 0 & 0 \\
O & \vdots & & \\
& \vdots & 0 & 0
\end{array}\right)
$$

with respect to the frame $\left\{E_{a}, F E_{a}, U_{\imath}, V_{i}\right\}$ in $O_{\imath}$, where $\Phi_{\imath}^{\prime}$ is a nonsingular real skew-symmetric $(4 m, 4 m)$-matrix. By Lemma $2.3 \Phi_{\imath}^{\prime}$ can be written in the form

$$
\Phi_{\imath}^{\prime}=\alpha_{\imath}^{\prime} \cdot \beta_{\imath}^{\prime}
$$

with $\alpha_{i}^{\prime} \in O(4 m)$ and $\beta_{i}^{\prime} \in H(4 m)$. If we put 


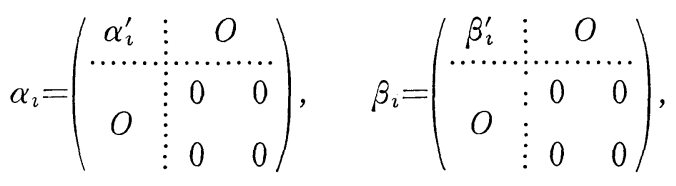

then $\alpha_{\imath}$ and $\beta_{\imath}$ define tensor fields of class $C^{\infty}$ in $O_{\imath}$. Since $\Phi_{\imath}^{\prime}$ is skew-symmetric,

and hence

$$
\beta_{i}^{\prime} \cdot{ }^{t} \alpha_{i}^{\prime}=-\alpha_{i}^{\prime} \cdot \beta_{i}^{\prime}
$$

$$
\beta_{\imath}^{\prime}=-\alpha_{\imath}^{\prime} \cdot \beta_{\imath}^{\prime} \cdot \alpha_{\imath}^{\prime}=-\alpha_{\imath}^{\prime 2}{ }^{t} \alpha_{\imath} \beta_{\imath}^{\prime} \alpha_{\imath}^{\prime} .
$$

As is easily seen, $-\alpha_{\imath}^{\prime 2} \in O(4 m),{ }^{t} \alpha_{\imath}^{\prime} \cdot \beta_{\imath}^{\prime} \cdot \alpha_{\imath}^{\prime} \in H(4 m)$. Thus, by the uniqueness of the decomposition, we obtain

$$
\begin{aligned}
& \alpha_{\imath}^{\prime 2}=-I_{4 m}, \\
& { }^{t} \alpha_{\imath}^{\prime} \cdot \beta_{\imath}^{\prime} \cdot \alpha_{i}^{\prime}=\beta_{\imath}^{\prime}, \text { i. e. } \beta_{\imath}^{\prime} \cdot \alpha_{\imath}^{\prime}=\alpha_{\imath}^{\prime} \cdot \beta_{i},
\end{aligned}
$$

$I_{4 m}$ being the unit $(4 m, 4 m)$-matrix. Consequently, we have

$$
\Phi_{\imath}=\alpha_{\imath} \cdot \beta_{i}
$$

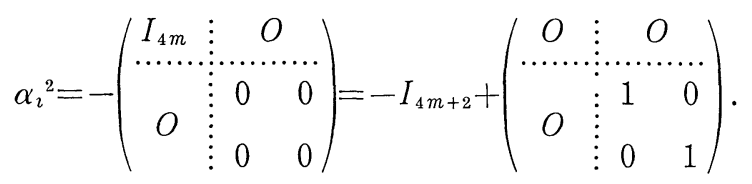

On the other hand, the complex structure $F$ has components of the form

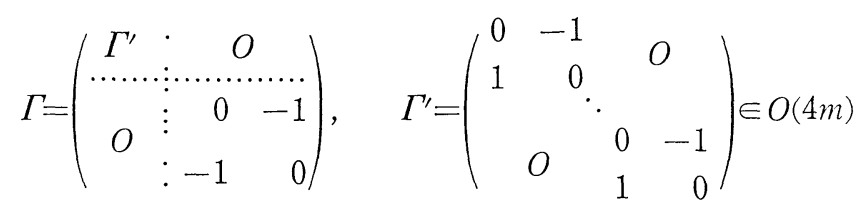

with respect to the adapted frame $\left\{E_{a}, F E_{a}, U_{\imath}, V_{i}\right\}$ in $O_{\imath}$. Hence Lemma 2.2 implies that $\hat{H}_{\imath}$ has components of the form

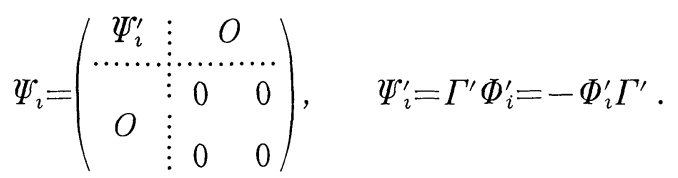

Therefore $\Psi_{\imath}^{\prime}$ can be decomposed as

$$
\Psi_{\imath}^{\prime}=\left(\Gamma^{\prime} \cdot \alpha_{\imath}^{\prime}\right) \cdot \beta_{\imath}^{\prime}=\delta_{\imath}^{\prime} \cdot \beta_{\imath}^{\prime}, \quad \delta_{\imath}^{\prime}=\Gamma^{\prime} \cdot \alpha_{\imath}^{\prime} .
$$

Denote by $\gamma_{\imath}$, the transformation of adapted frames $\left\{E_{a}, F E_{a}, U_{\imath}, V_{i}\right\}$ and $\left\{E_{a}^{\prime}, F E_{a}^{\prime}, U_{\jmath}, V_{\jmath}\right\}$ in $O_{i} \cap O_{\jmath}$. Then Lemmas 2.2 and 2.4 imply 


$$
\Phi_{\imath}=\gamma_{\imath \jmath} \cdot\left(a \Phi_{j}-b \Psi_{\jmath}\right) \cdot t \gamma_{\imath \jmath} .
$$

Substituting (3.5) and (3.9) into this, we have

$$
\begin{aligned}
\alpha_{\imath}^{\prime} \cdot \beta_{\imath}^{\prime} & =\gamma_{i j}^{\prime} \cdot\left(a \alpha_{j}^{\prime}-b \delta_{\jmath}^{\prime}\right) \cdot \beta_{j}^{\prime} \cdot t \gamma_{\imath \jmath}^{\prime} \\
& =\left(\gamma_{\imath \jmath}^{\prime} \cdot\left(a \alpha_{j}^{\prime}-b \delta_{\jmath}^{\prime}\right) \cdot{ }^{t} \gamma_{\imath \jmath}^{\prime}\right) \cdot\left(\gamma_{2 \jmath}^{\prime} \cdot \beta_{j}^{\prime} \cdot{ }^{t} \gamma_{\imath \jmath}^{\prime}\right),
\end{aligned}
$$

where $\gamma_{\imath 2}^{\prime}$ denotes the element of $O(4 m)$ such that

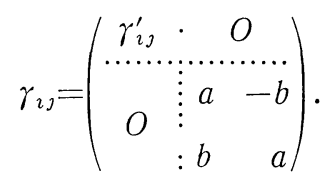

Since $\gamma^{\prime}{ }_{i j} \in O(4 m)$ and the decomposition is unique, we get

$$
\begin{aligned}
& \alpha_{\imath}^{\prime}=\gamma_{\imath \jmath}^{\prime} \cdot\left(a \alpha_{\jmath}^{\prime}-b \delta_{\jmath}^{\prime}\right) \cdot{ }^{t} \gamma_{\imath \jmath}^{\prime}, \\
& \beta_{\imath}^{\prime}=\gamma_{\imath j}^{\prime} \cdot \beta_{j}^{\prime} \cdot{ }^{t} \gamma_{\imath \jmath}^{\prime} .
\end{aligned}
$$

The equation (3.11) shows by means of Lemma 2.4 that $\left\{\beta_{i}\right\}$ defines a global tensor field $g$ of class $C^{\infty}$, which is a Hermitian metric in $M$.

Denote by $G_{\imath}$ the local tensor field of type $(1,1)$ having components $\alpha_{\imath}$ with respect to the adapted frame $\left\{E_{a}, F E_{a}, U_{\imath}, V_{i}\right\}$ in $O_{\imath}$. Thus (3.4) implies

$$
G_{i} U_{\imath}=G_{\imath} V_{\imath}=0, \quad u_{i} \circ G_{\imath}=v_{i} \circ G_{\imath}=0 \quad \text { in } O_{\imath}
$$

and (3.6) implies

$$
G_{\imath}{ }^{2}=-I+u_{\imath} \otimes U_{i}+v_{i} \otimes V_{\imath} \quad \text { in } O_{\imath} .
$$

Furthermore, using (3.3) and (3.4), we have

$$
\begin{aligned}
& g\left(G_{\imath} X, Y\right)=\hat{G}_{\imath}(X, Y) \\
& g\left(U_{\imath}, X\right)=u_{\imath}(X), \quad g\left(V_{\imath}, X\right)=v_{\imath}(X)
\end{aligned}
$$

for any vector fields $X$ and $Y$.

Next, we denote by $H_{2}$ the local tensor field of type $(1,1)$ having components $\Gamma \cdot \alpha_{\imath}$ with respect to the adapted frame $\left\{E_{a}, F E_{a}, U_{\imath}, V_{\imath}\right\}$ in $O_{\imath}$. Then

$$
H_{\imath}=F G_{\imath} \quad \text { in } \quad O_{\imath}
$$

holds. Then (3.10) implies

$$
G_{\imath}=a G_{j}-b H_{\jmath}, \quad H_{\imath}=b G_{\jmath}+a H_{\jmath}, \quad \text { in } O_{\imath} \cap O_{\jmath}
$$

Summing up Lemma 3.1, (3.12), (3.13), (3.15) and (3.16), we see that $\left\{\left(u_{\imath}, U_{\imath}\right.\right.$, $\left.\left.G_{\imath}, O_{\imath}\right): O_{\imath} \in \mathfrak{X}\right\}$ is a complex almost contact structure in $M$. Thus the Theorem is proved. 


\section{BIBLIOGRAPHY}

[1] Chevalley., C., Theory of Lie groups I, Princeton Univ. Press, 1946.

[2] Hatakeyama, Y., On the existence of Riemannian metrics associated with 2-form of rank 2r, Tôhoku Math. J. 14 (1962), 162-166.

[3] Ishinara, S. and M. Konish1, Real contact 3-structure and complex contact structure, Southeast Asian Bulletin of Math., 3 (1979), 151-161.

[4] Shibuya, Y., On the existence of a complex almost contact structure, Koda1 Math. J. 1 (1978) 197-204.

Tokyo Institute of Technology 